\title{
Variability in yield traits of TILLING population of bread wheat (Triticum aestivum L.)
}

\section{Rajender Singh*, Ratan Tiwari, Davinder Sharma, Vinod Tiwari and Indu Sharma}

ICAR- Indian Institute of Wheat and Barley Research, Karnal- 132001 (Haryana), INDIA

*Corresponding author. E-mail: rajenderkhokhar@yahoo.com

Received: January 18, 2015; Revised received: May 2, 2015; Accepted: May 29, 2015

Abstract: Mutagenesis is one of the powerful genetic strategies for crop improvement programmes. A chemically induced mutated genetic resource for detecting novel variations by Targeting Induced Local Lesions IN Genomes (TILLING) has been developed in recently released bread wheat (Triticum aestivum) cultivar DPW621-50. A total of $3,478 \mathrm{M}_{2}$ plants were evaluated for plant height, number of tillers/plant, thousand grain weight, number of seeds/ spike and grain yield/plant. A large variation was observed for all the traits. The highest frequency $(52.2 \%)$ of lines had similar height between $91-100 \mathrm{~cm}$ to the non-mutagenized DPW $621-50$ control followed by $28.9 \%$ of lines with height between $81-90 \mathrm{~cm}$. A large variation was observed in number of tillers/plant which ranged from 1-35 tillers/ plant. The highest frequency (32.09\%) lines had $31-40$ seeds/spike followed by $29.84 \%$ lines with $41-50$ seeds/ spike. Few lines $(0.35 \%)$ had more than 70 seeds/spike with normal seed size as their thousand grain weight ranged between 34.82-43.82g. Chlorophyll deficient, grassy type and sterile mutants were also observed. This population may serve as new genetic resource for functional genomics studies and novel variants for different traits in elite germplasm can be made available to the plant breeders for wheat improvement.

Keywords: Mutagenesis, TILLING, Triticum aestivum

\section{INTRODUCTION}

Exploiting natural or induced genetic variability is a proven strategy in the improvement of all major food crops including wheat. Mutagenesis is an important tool in crop improvement to generate a vast amount of genetic variability for identification of improved or novel phenotypes that can be exploited in conventional plant breeding programmes and genetic studies (Parry et al., 2009). Induced mutations through non transgenic technique called Targeting Induced Local Lesions IN Genomes (TILLING) are now being widely used for developing improved crop varieties and for the discovery of genes controlling important traits and understanding the functions and mechanisms of actions of these genes in wheat (Slade et al., 2005; Dong et al., 2009; Uauy et al., 2009; Feiz et al., 2009; Chen et al., 2012). The range of alleles can be developed in a short time is matchless and unlikely to be found elsewhere in the pool of germplasm accessible to plant breeders, including land races and undomesticated relatives. As the TILLING population is a stable source, the results of basic scientific research can be well interpreted into crop improvement as new information about the functions of potential gene targets becomes available. The application of TILLING to crop improvement may help with the constraint in domesticated species genome such as limited genetic variation. During domestication and subsequent selection, much of the genetic variation available in the wild crop progenitors has been lost (Gepts and Papa, 2002). Thus, plant breeders have at times used wild relatives or landraces to introduce useful genetic variation which was successful in wheat for developing disease resistant and higher yielding varieties. As an alternative to the use of wild varieties, TILLING can be a means to introduce genetic variation in an elite germplasm without the need to acquire variation from exotic cultivars, thus avoiding introduction of agriculturally undesirable traits (Slade and Knauf, 2005). The objective of the present work was to generate mutant population of wheat cultivar DPW621-50 using the chemical mutagen, ethyl methane sulphonate (EMS) and characterize them for a range of traits including plant height, number of tillers/plant, thousand grain weight, number of seeds/spike and grain yield/plant so that they can be availed to identify novel mutants and made use of in functional genomics for discovery of genes and novel alleles in wheat.

\section{MATERIALS AND METHODS}

The wheat cultivar DPW621-50 (KAUZ//ALTAR84/ AOS/3/MILAN/KAUZ/4/HUITES) released during 2010-11 for timely sown and irrigated conditions of North Western Plain Zone (NWPZ) of India was used to generate a mutant population for TILLING. Ten thousand seeds were soaked in $0.7 \%(\mathrm{v} / \mathrm{v})$ EMS 
solution (Sigma-Aldrich ${ }^{\circledR}$ ) with gentle agitation on a shaker at $75 \mathrm{rpm}$ overnight for $16 \mathrm{~h}$ at room temperature (Dong et al., 2009; Uauy et al., 2009)). After EMS treatment, the seeds were thoroughly washed under running tap water for $6 \mathrm{~h}$ and then sown in the field at ICAR- Indian Institute of Wheat and Barley Research, Karnal, India. Fertilizers, herbicides and irrigations were applied as per recommended package of practice for NWPZ. One spike from each $\mathrm{M}_{1}$ plant was harvested and a single seed from each spike was used to $\mathrm{M}_{2}$ plant population generate (Uauy et al., 2009; Parry et al., 2009). One seed from each $\mathrm{M}_{1}$ plant were grown to maturity and phenotyped for morphological and yield related traits such as plant height, tiller number/plant, seed number/spike and thousand grain weight. From each $\mathrm{M}_{2}$ plant, $\mathrm{M}_{3}$ seeds were collected and packed for future use.

\section{RESULTS AND DISCUSSION}
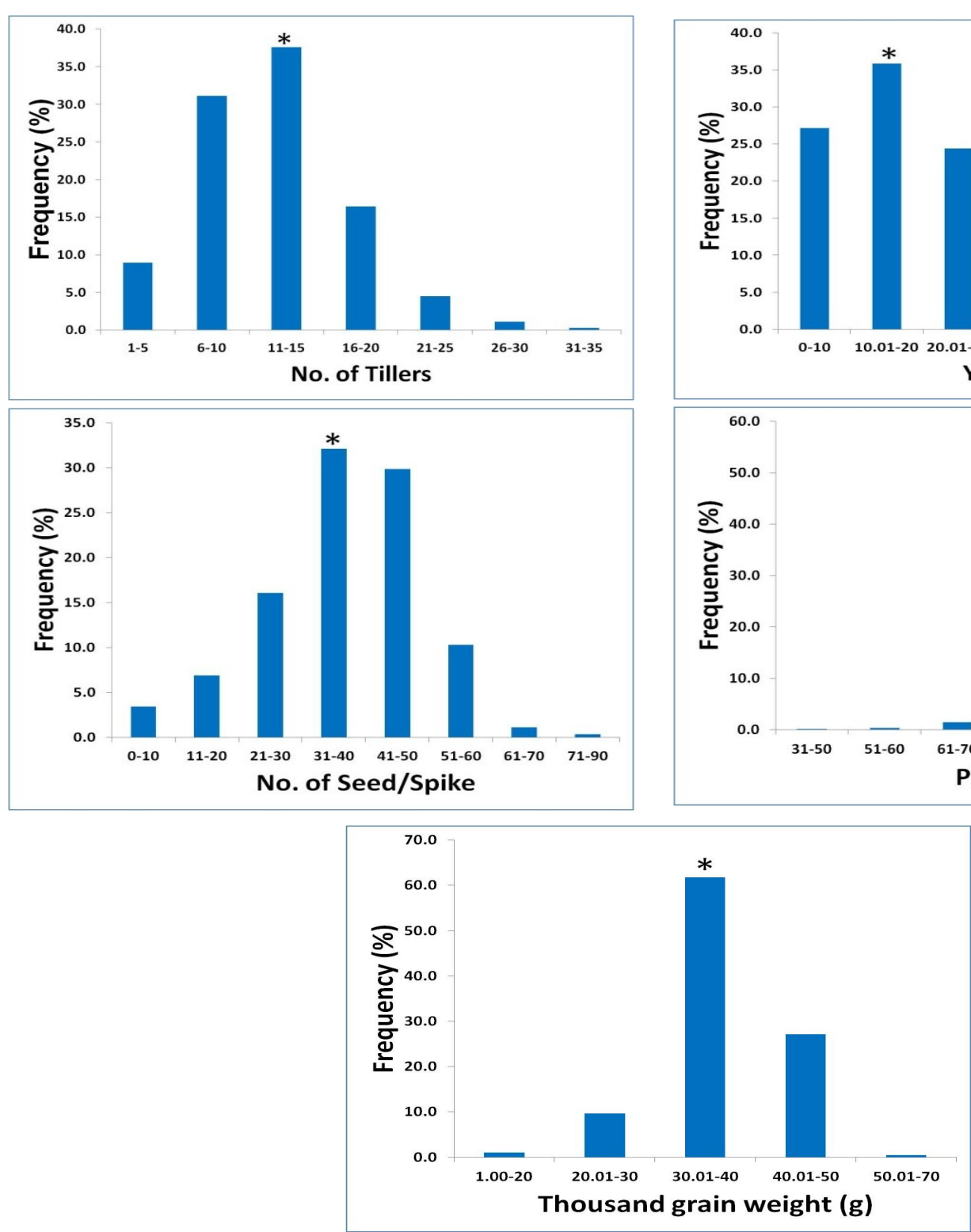

Fig. 1. Frequency distribution of $M_{2}$ population based on number of tillers/plant, number of seeds/spike, thousand grain weight, yield/plant and plant height. $(*$ indicate the parent genotype DPW621-50).
A total of 3,478 $\mathrm{M}_{2}$ plants were evaluated for phenotypic variations. The phenotypic screen included plant height, number of tillers/plant, thousand grain weight (TGW), number of seeds/spike and grain yield/plant. The mutagenized population showed extensive variability in morphological and agronomic traits and a range of phenotypes were observed in $\mathrm{M}_{2}$ population (Fig. 1). The highest frequency $(52.2 \%)$ of plants had similar height between $91-100 \mathrm{~cm}$ to the non-mutagenized DPW 621-50 (control) followed by $28.9 \%$ of plants with height between $81-90 \mathrm{~cm}$. Only $1.8 \%$ of plants had the plant height less than $70 \mathrm{~cm}$. The shortest and tallest plant had $31 \mathrm{~cm}$ and $115 \mathrm{~cm}$ height, respectively with mean plant height of the population was $92 \mathrm{~cm}$. A large variation was observed in number of tillers/plant which ranged from 1-35 tillers/plant with a mean of 12 tillers/plant. The highest frequency $(37.6 \%)$ of plants had 11-15 tillers/plant which was similar to control genotype. $22.3 \%$ of plants

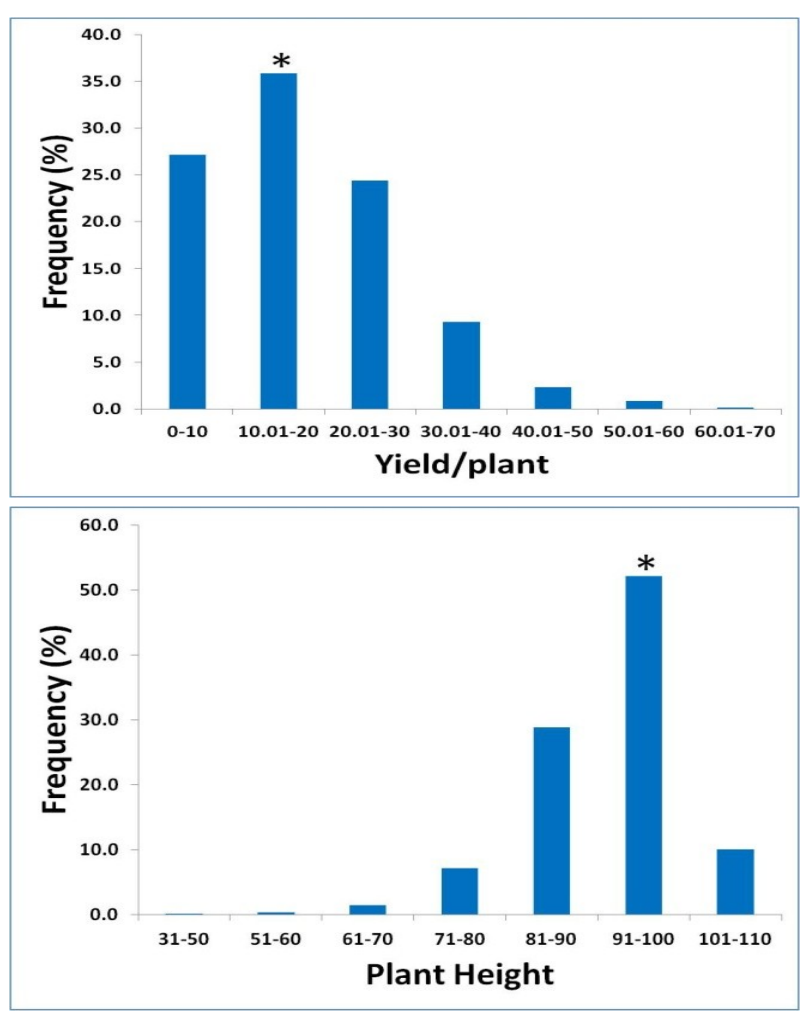




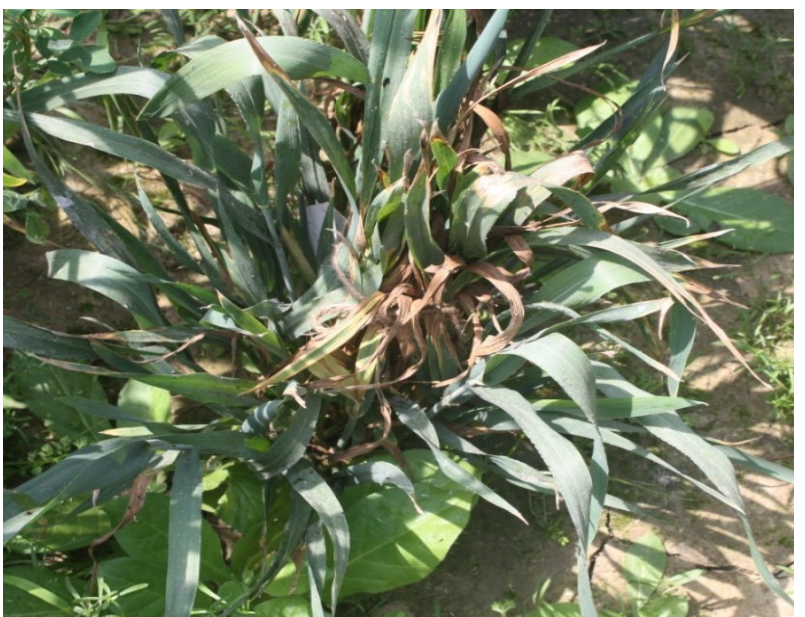

Grassy mutant

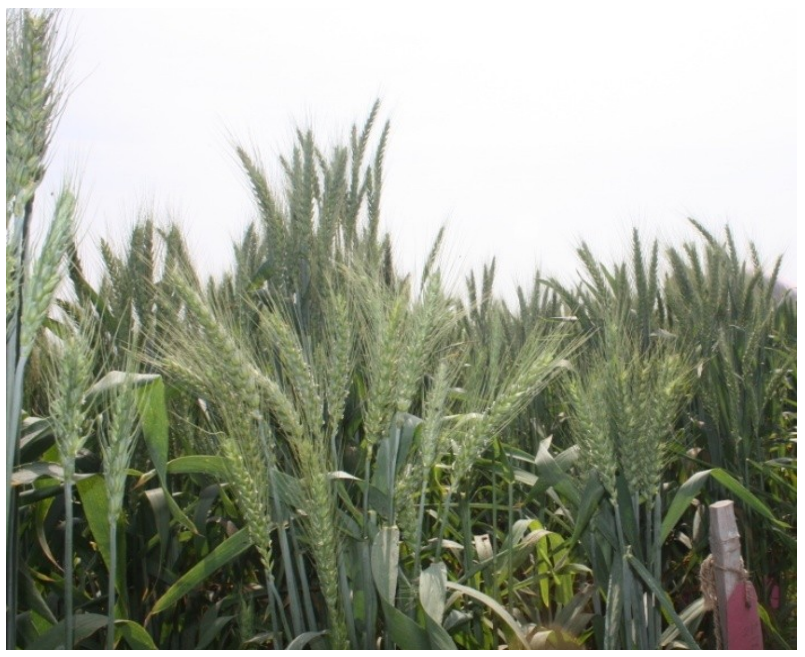

Plant height variation

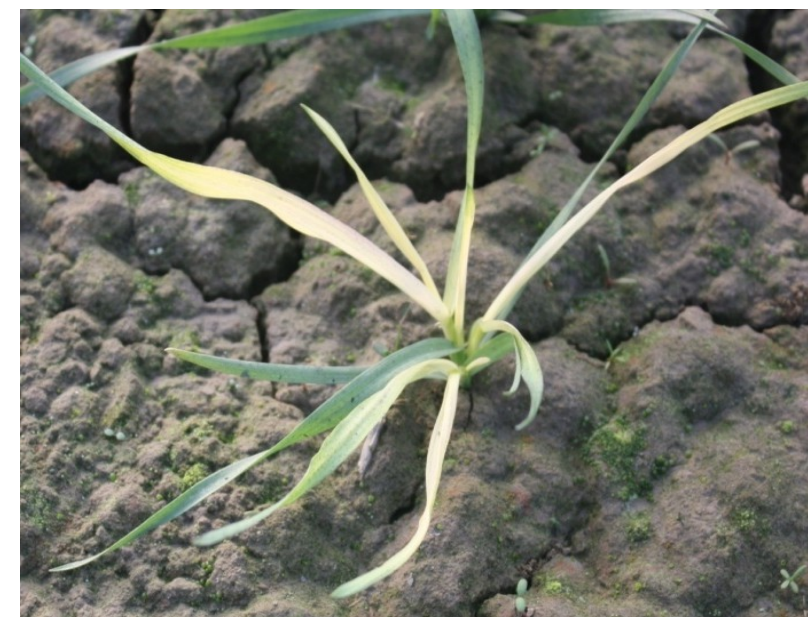

Chlorophyll deficient mutant

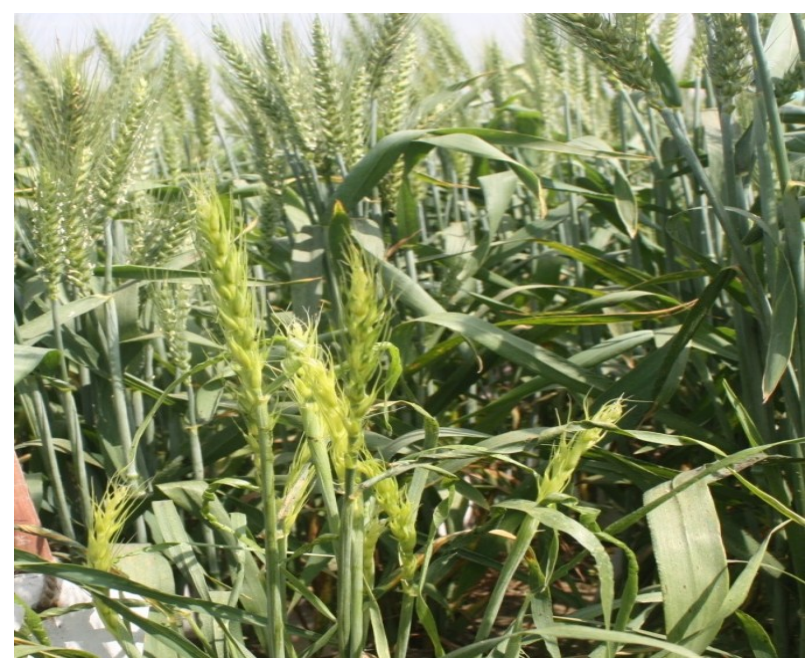

Sterility

Fig. 2. Mutants observed in $M_{2}$ population.

had more number of tillers/plant than DPW621-50. Chen et al. (2012) also observed the wide range of the phenotypic variations in plant height, heading date and number of tillers and seed size in hexaploid wheat.

The mean thousand grain weight of the population was 36.73 g. Majority $(61.79 \%)$ of plants has TGW in the range of $30-40 \mathrm{~g}$ along with control. TGW of $27.51 \%$ of $\mathrm{M}_{2}$ plants was more than $40 \mathrm{~g}$.A large variability was also observed for number of seeds/spike in the mutagenized population. Majority $(32.09 \%)$ of plants had $31-40$ seeds/spike followed by $29.85 \%$ plants having 41-50 seeds/plants along with control. Complete and partial sterility was also observed in few plants and $3.39 \%$ of plants have less than 10 seeds/ spike. Few plants (3.8\%) had more than 50 seeds/spike having TGW more than $40 \mathrm{~g}$. The highest frequency of plants $(35.9 \%)$ had grain yield/plant between 10.01 $-20.00 \mathrm{~g}$ followed by $24.4 \%$ plants having grain yield/ plant between 20.01-30 g. Some plants (3.2\%) had more than $40 \mathrm{~g}$ yield and $6.17 \%$ plants had yield less than $2 \mathrm{~g}$ representing low fertility in the mutagenized population. Low fertility plant at a frequency of $0.19 \%$ in hexapoid wheat (Chen et al., 2012) and 4.5\% sterile plant have been observed in diploid wheat (Rawat et al., 2012). Two chlorophyll deficient mutants were also observed in seedling stage but further these plants did not survive. Three grassy types of mutants were also observed which did not flower (Fig. 2). Phenotypic mutants, such as albino, dwarf, grassy-shoot, broad leaf, early/late flowering, and delayed senescence has been observed in the $\mathrm{M}_{2}$ generation of EMS induced mutant populations of wheat (Chen et al., 2012; Rawat et al., 2012).

The primary objective of this effort was to generate an EMS induced mutant population in well adapted Indian wheat cultivar DPW621-50 for various morphological, developmental and yield related traits as well as for biotic and abiotic stress tolerance. TILLING has been widely used in wheat for manipulation of starch composition (Slade et al., 2005; Dong et al., 2009; Slade et al., 2012), grain hardness (Dong et al., 2009), high molecular weight gluten subunits (HMW-GS) composition (Laudencia-Chingcuanco, 2012), phytic acid level (Guttieri et al., 2004), disease resistance 
(Campbell et al., 2012), vernalization (Chen and Dubcovsky, 2012) and seed dormancy (Schramm et al., 2012).

Mutagenesis offers a distinct advantage of being a non-transgenic alternative for rapid generation of novel genetic variation which can provide a rich and convenient resource for functional genomics and also for conventional cop improvement programmes as desired mutant alleles can be easily introgressed into different genetic backgrounds or the mutants themselves can be developed in advanced genetic materials for commercial use. The range of alleles that can be discovered via TILLING to develop new traits is unparalleled, and unlikely to be found elsewhere in the pool of germplasm including landraces and wild relatives accessible to plant breeders (Slade et al., 2012).

\section{Conclusion}

The variability observed in plant height, no.of tillers/ plant, thousand grain weight and no. of seeds/spike in the mutagenized wheat population described here suggests that it will be a useful resource for forward and reverse genetic studies and also for wheat improvement through conventional plant breeding. The mutant lines having high grain number per spike $(>70$ seeds/spike) along with desirable thousand grain number (34.82-43.82g) can be exploited for yield enhancement. The mutant population can also be phenotyped for tolerance to biotic and abiotic stresses, nutritional quality, resource use efficiency etc. for wheat improvement.

\section{REFERENCES}

Campbell, J., Zhang, H., Giroux, M.J., Feiz, L., Jin, Y., Wang, M., Chen, X. and Huang, L. (2012). Amutagenesis -derived broad-spectrum disease resistance locus in wheat. Theoretical and Applied Genetics, 125:391-404.

Chen, A. and Dubcovsky, J. (2012). Wheat TILLING mutants show that the vernalization gene VRN1 down-regulates the flowering repressor $V R N 2$ in leaves but is not essential for flowering. Plos Genetics, 8: e1003134.

Chen, L., Huang, L., Min, D., Phillip, A., Wang, S., Madgwick, P.J., Parry, M.A. and Hu, Y.G. (2012).
Development and characterization of a new TILLING population of common bread wheat (Triticum aestivum L). PLos ONE 7(7): e41570.

Dong, C., Dalton-Morgan, J., Vincent, K. and Sharp, P. (2009). A modified TILLING method for wheat breeding. Plant Genome, 2:39-47.

Feiz, L., Beecher, B.S., Martin, JM. and Giroux, M.J. (2009). In planta mutagenesis determines the functional regions of the wheat puroindoline proteins. Genome, 183:853-860.

Gepts, P. and Papa, R. (2002). Evolution during domestication. Encyclopedia of Life Sciences, :1-7.

Guttieri, M., Bowen, D., Dorsch, J.A., Raboy, V. and Souza, E. (2004). Identification and characterization of a low phytic acid wheat. Crop Science, 44:418-424

Laudencia-Chingcuano, D. (2012). Isolation and characterization of EMS-induced Dy10 and Ax1 high molecular weight glutenin subunit deficient mutant line of elite hexaloid wheat (Triticum aestivum L.) cv Summit. Journal of Cereal Science, 56:296-299.

Parry, M.A.J., Madgwick, P.J., Bayon, C., Tearall, K., Hernandez-Lopez, A., Baudo, M., Rakszegi, M., Hamada, W., Al-Yassin, A., Ouabbou, H., Labhilili, M. and Phillips, A.L. (2009). Mutation discovery for crop improvement. Journal of Experimental Botany, 60: 2817-2825.

Rawat, N., Sehgal, S.K., Joshi, A., Rothe, N., Wilson, D.L., McGraw, N., Vadlani, P.V., Li, W. and Gill, B.S. (2012). A diploid wheat TILLING resource for wheat functional genomics. BMC Plant Biology, 12:205.

Schramm, E.C., Nelson, S.K. and Steber, C.M. (2012). Wheat ABA-insenstive mutants result in reduced grain dormancy. Euphytica, 188:35-49.

Slade, A.J., Fuerstenberg, S.I., Loeffler, D., Steine, M.N. and Facciotti, D. (2005). A reverse genetic, nontransgenic approach to whet improvement by TILLING. Nature Biotechnology, 23:75-81.

Slade, A.J. and Knauf V.C. (2005). TILLING moves beyond functional genomics into crop improvement. Transgenic Research, 14:109-115.

Slade, A.J., McGuire, C., Loeffler, D., Mullenberg, J., Skinner, W., Fazio, G., Holm, A., Brandt, K.M., Steine, M.N., Goodstal, J.F. and Knauf, V.C. (2012). Development of high amylose wheat through TILLING. BMC Plant Biology, 12:69.

Uauy, C., Paraiso, F., Colasuonno, P., Tran, R.K., Tsai, H., Berardi, S., Comai, L. and Dubcovsky, J. (2009). A modified TILLING approach to detect induced mutations in tetraploid and hexaploid wheat. BMC Plant Biology, 9:115. 